\title{
HERRAMIENTAS DE ERGONOMÍA EN LOS SISTEMAS DE SALUD. ALTERNATIVAS PARA EL MEJORAMIENTO DE LAS TAREAS Y LA TECNOLOGÍA
}

\author{
ERGONOMICS TOOLS IN HEALTH SYSTEMS. ALTERNATIVES FOR TASK AND \\ TECHNOLOGY IMPROVEMENT
}

\author{
John Alexander Rey-Galindo* \\ Alber Eduardo Duque-Álvarez ${ }^{* *}$
}

\begin{abstract}
Resumen: Extender y fortalecer la prestación del servicio de salud, resulta imperativo dentro de los propósitos de mejoramiento de la calidad de vida de la población. Modelos como el Systems Engineering Initiative for Patient Safety (SEIPS), desde la ergonomía/factores humanos han contribuido a identificar y comprender los componentes de los sistemas de salud y la manera en cómo están relacionados. No obstante, en la búsqueda por hacer que en Latinoamérica, la presencia de la ergonomía/factores humanos se fortalezca en los sistemas de salud, es necesario, además, evidenciar algunas de las herramientas que pueden constituirse en alternativas muy importantes para encontrar avances en el funcionamiento de estos sistemas y con ello del bienestar de la población. Para ello, en el presente texto se describen seis herramientas que pueden ser usadas para la evaluación de las tareas y de los recursos tecnológicos; dos áreas descritas desde el modelo SEIPS 3.o. Se espera con esto, contribuir a la comprensión metodológica de la ergonomía/factores humanos, para su integración más clara en los procesos de mejora de los sistemas de salud.
\end{abstract}

Palabras clave: Sistemas de salud, ergonomía/factores humanos, evaluación de tareas, evaluación de tecnología.

\begin{abstract}
To improve the population's quality of life, it is imperative to extend and strengthen the health services provision. Models such as the Systems Engineering Initiative for Patient Safety (SEIPS), from ergonomics/human factors, have contributed to identify and understand the health systems components and how they are related. However, it is also necessary to highlight some of the tools to strengthen the ergonomics/human factors presence in Latin America's health systems and contribute to improving the well-being of the population. For this purpose, this text describes six tools that can be used for assessing tasks and technology. Those areas are defined based on the SEIPS 3.0 model. It is hoped that this will contribute to the methodological understanding of ergonomics/human factors for their more direct integration into the improvement processes of health systems.
\end{abstract}

Keywords: Healthcare systems, ergonomics/human factors, tasks assessment, technology assessment.

*Centro de Investigaciones en Ergonomía, Universidad de Guadalajara. Guadalajara, México. Correo electrónico: john.rey@academicos.udg.mx. Orcid: https://orcid.org/oooo-0002-0458-7107. Autor de correspondencia.

**Universidad de Guadalajara. Guadalajara, México. Correo electrónico: alberduque91@gmail.com. Orcid: https://orcid.org/oooo-0001-6427-2142 
Recepción: 15.06.2021 / Revisión: 23.06.2021 / Aceptación: 29.07.2021

\section{Introducción}

Extender y fortalecer la prestación del servicio de salud, resulta imperativo dentro de los propósitos de mejoramiento de la calidad de vida de la población. En esta búsqueda, desde hace varias décadas, se ha identificado la necesidad de trabajar en el robustecimiento de los sistemas prestadores del servicio de salud, no solo incrementando su capacidad de cobertura, sino también la calidad y seguridad con la que realizan sus actividades (UNICEF \& World Health Organization [WHO], 1978; WHO, 2003).

Con el paso del tiempo se han reconocido problemas fundamentales. Aspectos como malas praxis o errores médicos de distinta índole (Al-Assaf et al., 2003), relacionados con atención primaria y ambulatoria, errores de medicación, aspectos de seguridad, entre otros, resultan comunes. Lo anterior con incidencias en Latinoamérica de entre el $8 \%$ y el 20\% (WHO, 2019; Lavanderos et al., 2016), y la mayoría de estos problemas con posibilidad de haber sido prevenidos.

Ante esta circunstancia, la ergonomía/factores humanos (E/FH) ha encontrado un espacio como mecanismo de análisis y comprensión de los aspectos que impactan a los servicios de salud. Su naturaleza interdisciplinar y su perspectiva sistémica, le permiten un acercamiento a las generalidades y particularidades de los sistemas de salud en el reconocimiento y proceso de mejora de diversos aspectos teniendo como centro el bienestar del paciente.

Lo anterior se evidencia en el Global Patient Safety Action Plan 2021-2030 (Third Draft) (WHO, 2021) en su segundo objetivo estratégico, donde se establece la necesidad de incorporar la perspectiva de la $\mathrm{E} / \mathrm{FH}$, dentro de los sistemas de salud, para mejorar y fortalecer la estructura de dichos sistemas, su resiliencia y las formas de operación, partiendo del elemento esencial que es el cuidado diario del paciente.

Cabe señalar que la integración de la E/FH como parte importante de los sistemas de salud no es reciente. Desde la década de 1970 se trabajó en su inclusión, con un enfoque desde el ámbito de la seguridad de los trabajadores y del análisis de los objetos y espacios (Singleton, 1972). No obstante, el sentido en el que actualmente se está reconociendo, es definitivamente más amplio y responde a su naturaleza centrada en el usuario, en este caso, centrada en el bienestar del paciente (Carayon et al., 2014; WHO \& Patient-Safety, 2009).

En el proceso de integración de la $\mathrm{E} / \mathrm{FH}$ dentro de los sistemas de salud, se ha identificado la necesidad de ir construyendo formas de análisis e intervención más acordes con los requerimientos propios de estos escenarios (Jun et al., 2009). Uno de los modelos que más ha contribuido a dilucidar desde la ergonomía, los aspectos a considerar dentro de estos sistemas es el modelo Systems Engineering Initiative for Patient Safety (SEIPS) (Carayon et al., 2006).

En sus tres versiones, el SEIPS nos acerca a la estructura socio-técnica de los sistemas de salud, identificando distintos entornos, momentos y relaciones entre los componentes, en pro del bienestar del paciente (Carayon et al., 2020). No obstante, su característica 
esencialmente descriptiva y general, considerando sus tres versiones en cada uno de sus enfoques (Carayon et al., 2020; Holden et al., 2013; Carayon et al., 2006), hace necesaria una mirada más específica.

Bajo la anterior idea, el presente texto tiene como propósito describir métodos y herramientas del ámbito de la $\mathrm{E} / \mathrm{FH}$, que pueden ser considerados para analizar las tareas, y las herramientas y tecnología, componentes esenciales alrededor del cuidado del paciente, que establece el modelo SEIPS 3.0. Se considera que la identificación de dichas herramientas puede constituirse en un marco de referencia para el trabajo práctico desde la ergonomía dentro de los sistemas de salud.

\section{Materiales y métodos}

Partiendo de las tareas, y herramientas y tecnologías, áreas planteadas en el modelo SEIPS (Carayon et al., 2006), se definieron un conjunto de alternativas desde la ergonomía/factores humanos o relacionadas con sus fundamentos, que se pueden constituir en herramientas metodológicas para mejorar tanto las tareas dentro de los sistemas de salud, como la tecnología usada en este contexto. Estas herramientas metodológicas fueron elegidas considerando su alta versatilidad, su facilidad de aplicación en diversos contextos y su importancia en el desarrollo de sistemas centrados en el ser humano.

\section{Resultados y discusión}

\section{Tareas}

Cualquier actividad humana que involucre propósitos específicos, puede ser descrita como un conjunto de pasos o tareas que se llevan a cabo de manera secuencial o no secuencial a través de los cuales se obtiene el objetivo que se persigue. Esta manera de ver las actividades humanas ha dado paso a diversos métodos de análisis que han permitido entender de manera más clara la naturaleza de la que se componen. En el caso de los sistemas de salud, la evaluación de las tareas puede dar un panorama claro de los componentes que intervienen en distintos momentos del funcionamiento del sistema, pero además de la manera en cómo estos interactúan, lo que resulta esencial cuando se trata de analizar el contexto desde la perspectiva de E/FH. En este sentido, a continuación, se describen tres formas de estudiar tareas, que pueden ser usadas en el proceso de evaluación de un sistema de salud.

\section{Análisis jerárquico de tareas (Hierarchical Task Analysis)}

El análisis de tareas se enfoca en identificar y describir los pasos que se llevan a cabo en una actividad. Este tipo de análisis permite entender de manera detallada cada uno de los momentos que componen una tarea y los elementos y recursos que intervienen. La posibilidad de desglosar e identificar las sub-tareas o componentes evidencia también la identificación de las habilidades y conocimientos que se requieren en la realización de la actividad (Ruck, 1980). 
Dentro de la E/FH, el HTA (por sus siglas en inglés), ha tenido un espacio central. Este método específico, permite comprender y describir la estructura de la tarea a partir de su enfoque en los objetivos que la componen. En este sentido, se reconoce a la tarea como un conjunto de pasos que se dan desde sub-objetivos, todos ellos dirigidos a dar cuenta del objetivo general que determina la realización de la tarea (Salmon et al., 2010).

Stanton (2006) sostiene que la base del HTA se soporta en tres pilares específicos que son: a) Las tareas en un sentido más amplio son operaciones, que a su vez están determinadas por el objetivo del sistema. Por lo tanto, el análisis se enfoca en los objetivos del sistema. b) Todas las operaciones pueden ser sub-divididas, por lo que se encontrarán sub-objetivos. Dichos sub-objetivos deben poder ser evaluados y ponderados, en relación al objetivo central del sistema. c) Se debe considerar la relación entre las sub-operaciones y las posibles secuencias existentes. No obstante, es importante considerar que si bien la jerarquía de las sub-operaciones será esencial, la naturaleza de muchas de las actividades escapa de una secuencia y subordinación entre sub-operaciones.

El resultado de un HTA es una descripción de la operación o tarea, en donde se identifican los componentes, las acciones y las relaciones que existen entre cada sub-objetivo. Esta forma de análisis resulta muy útil para entender las condiciones y componentes del trabajo de las distintas formas de interacción entre los involucrados y demás componentes, dentro de un sistema de salud. El HTA puede ser aplicado a escenarios diversos dentro del contexto de la salud, desde tareas de carácter administrativo, pasando por el análisis de atención de enfermedades o padecimientos, hasta intervenciones quirúrgicas específicas.

Para llevar a cabo un HTA, la observación es la herramienta más útil. Una observación estructurada permite identificar aspectos como el orden o jerarquía de las tareas, los recursos que se usan, los requerimientos y demás elementos que entran en juego en la consecución de los objetivos (tabla 1). Además de la observación, otros formas de recabar información son los cuestionarios, las entrevistas a los expertos en las tareas, pruebas de usuario y revisión de documentación (Salmon et al., 2010).

Tabla 1. Ejemplo tomado de Sarker et al. (2008) donde se muestra la descripción de una tarea.

\begin{tabular}{|c|c|c|c|c|c|}
\hline No & Tarea & Plan & No & Sub-tareas & Recuperación \\
\hline \multirow[t]{4}{*}{5} & $\begin{array}{l}\text { Abrir } \\
\text { canal } \\
\text { inguinal }\end{array}$ & $\begin{array}{l}\text { Sub-tareas } 5.1 \text {, } \\
5.2,5 \cdot 3,5 \cdot 4 \text { en } \\
\text { orden consecutivo }\end{array}$ & 5.1 & $\begin{array}{l}\text { Pequeña incisión transversal } \\
\text { en la aponeurosis oblicua } \\
\text { externa, en línea con el anillo } \\
\text { externo }\end{array}$ & \\
\hline & & & 5.2 & $\begin{array}{l}\text { Incisión extendida lateral y } \\
\text { medialmente, soltando anillo } \\
\text { externo }\end{array}$ & \\
\hline & & & $5 \cdot 3$ & $\begin{array}{l}\text { Hojas superiores e inferiores } \\
\text { diseccionadas y pinzas } \\
\text { aplicadas a cada hoja, } \\
\text { formando bolsa lateral }\end{array}$ & \\
\hline & & & 5.4 & $\begin{array}{l}\text { Retractor transversal } \\
\text { insertado entre las hojas } \\
\text { superior e inferior }\end{array}$ & $\begin{array}{l}\text { El retractor de } \\
\text { Travers se sale, } \\
\text { vuelva a aplicar el } \\
\text { retractor }\end{array}$ \\
\hline
\end{tabular}


Es importante mencionar que una vez que se comprende la estructura de la tarea, pueden surgir otro tipo de análisis; por ejemplo, por cada sub-objetivo se podrían describir los recursos que se usan, un registro de errores de distinta índole (Lane et al., 2006), conocimiento requerido, tiempo de realización de la tarea y sub-tareas, entre otras diversas opciones que deben ser definidas desde los propósitos del análisis. En la figura 1 se muestran posibles aspectos a analizar a partir del uso del HTA.

Figura 1. Elementos a analizar en un HTA.

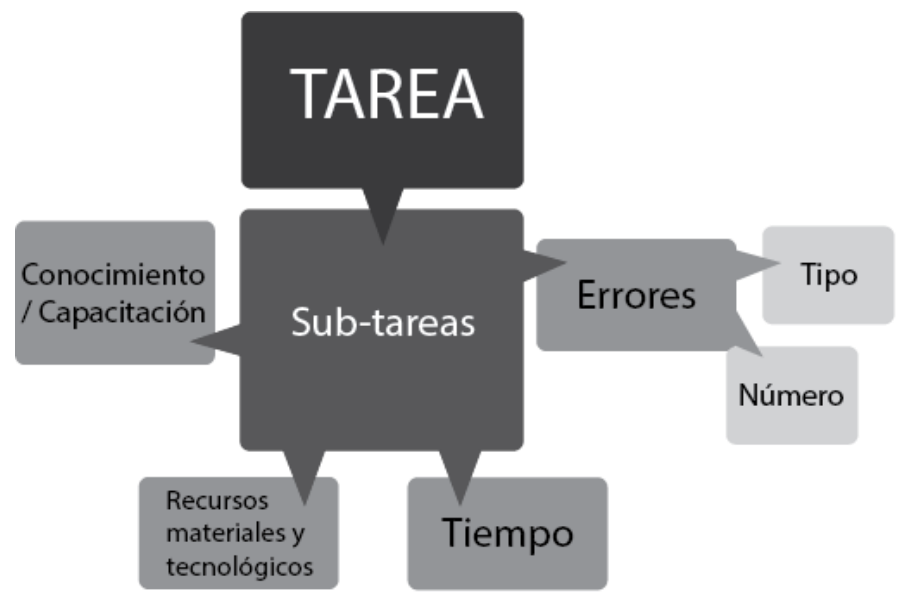

El HTA, desde su naturaleza dirigida a analizar el componente del trabajo en relación a sus objetivos y considerando su relativa simplicidad para ser realizado (Lane et al., 2006; Sarker et al., 2008), ha hecho que sea una herramienta ya usada en el contexto de la salud y se constituye en una muy buena opción de análisis en contextos como el latinoamericano, en donde los sistemas, por diversas razones de carácter social, cultural y económico, requieren de una revisión particular.

\section{Análisis cognitivo de tareas (Cognitive Task Analysis)}

Otro tipo de análisis enfocado en las tareas, usado en el ámbito de la ergonomía es el Análisis Cognitivo de Tareas o CTA (por sus siglas en inglés). Esta forma de acercamiento a lo que sucede a lo largo de la realización de las actividades se enfoca en identificar cuáles son los aspectos cognitivos presentes en las tareas, con el fin de reconocer aspectos como las demandas contenidas en ellas, los recursos cognitivos usados por los involucrados y posibles áreas de mejora (Potworowski \& Green, 2013) sustentadas en los conocimientos de los involucrados.

Esta forma de análisis, surge considerando la complejización de las tareas realizadas a partir de los nuevos recursos tecnológicos (Rasmussen, 1988). Si bien, en el caso del HTA entender lo que sucede en las tareas, en relación a los objetivos es lo esencial, en el caso del CTA el propósito está en relación a identificar las limitaciones de la interfaz (Hajdukiewicz \& Vicente, 2004), o de una manera más general, las limitaciones del conocimiento y la información presentes en el desempeño de las tareas.

Se podría afirmar que el CTA, más que un método específico, es un enfoque que permite acercarse a quien realiza la actividad, buscando entender sus marcos de referencia mentales. Clark et al. (2016) sostienen que, aunque existe una gran diversidad de formas de 
llevar a cabo un CTA, se pueden reconocer cinco fases generales que constituyen su estructura básica y para las cuales hay una cantidad igual de variada de herramientas en el proceso de aplicación (tabla 2).

Tabla 2. Pasos y herramientas para la aplicación de un CTA basado en lo planteado por Clark et al. (2016).

\begin{tabular}{|c|c|c|}
\hline $\begin{array}{l}\text { Fases generales } \\
\text { de un } C T A\end{array}$ & Descripción & Posibles herramientas \\
\hline $\begin{array}{l}\text { Recopilar } \\
\text { conocimientos } \\
\text { preliminares }\end{array}$ & $\begin{array}{l}\text { Se identifica el contenido general del } \\
\text { trabajo y la información presente. También } \\
\text { se suman expertos en las actividades, que } \\
\text { acompañen el proceso de análisis. }\end{array}$ & $\begin{array}{l}\text {-Análisis de documentos } \\
\text {-Observaciones } \\
\text {-Entrevistas no } \\
\text { estructuradas }\end{array}$ \\
\hline $\begin{array}{l}\text { Identificar } \\
\text { representaciones } \\
\text { del conocimiento }\end{array}$ & $\begin{array}{l}\text { Se describen las tareas en sub-tareas y se } \\
\text { identifica los modelos mentales y el } \\
\text { conocimiento de los involucrados para } \\
\text { realizarlas. }\end{array}$ & $\begin{array}{l}\text {-Mapas conceptuales } \\
\text {-Diagramas de flujo } \\
\text {-Diagrama de tareas } \\
\text {-Redes semánticas }\end{array}$ \\
\hline $\begin{array}{l}\text { Aplicar métodos de } \\
\text { obtención de } \\
\text { conocimiento } \\
\text { enfocado }\end{array}$ & $\begin{array}{l}\text { Se analiza el conocimiento que tienen } \\
\text { quienes realizan las actividades y la forma } \\
\text { de adquirirlo y aplicarlo. Esto permite } \\
\text { identificar problemáticas y la manera en la } \\
\text { que encaran dichas problemáticas. }\end{array}$ & $\begin{array}{l}\text {-Mapas conceptuales } \\
\text {-Auditoría de conocimientos } \\
\text { en equipo (TKA) } \\
\text {-Método conceptos, } \\
\text { procesos y principios (CPP) } \\
\text {-Método de decisión crítica } \\
\text { (CDM) }\end{array}$ \\
\hline $\begin{array}{l}\text { Analizar y verificar } \\
\text { la información } \\
\text { adquirida }\end{array}$ & $\begin{array}{l}\text { Se organizan, codifican y catalogan los } \\
\text { hallazgos, con el fin de ser analizados y } \\
\text { verificados por otros expertos. }\end{array}$ & $\begin{array}{l}\text {-Análisis de discurso } \\
\text {-Método conceptos, } \\
\text { procesos y principios (CPP) } \\
\text {-Método de Decisión Crítica } \\
\text { (CDM) }\end{array}$ \\
\hline $\begin{array}{l}\text { Ordenar los } \\
\text { resultados para } \\
\text { realizar una } \\
\text { aplicación y mejora }\end{array}$ & $\begin{array}{l}\text { Se desarrollan modelos de las tareas que } \\
\text { permitan identificar habilidades esenciales } \\
\text { para realizarlas, posibles problemas } \\
\text { presentes y estrategias para enfrentarlos. }\end{array}$ & $\begin{array}{l}\text {-Método Conceptos, } \\
\text { Procesos y Principios (CPP) } \\
\text {-Método de Decisión Crítica } \\
\text { (CDM) }\end{array}$ \\
\hline
\end{tabular}

Ya existen diversos estudios enfocados en el ámbito médico, donde se ha aplicado el enfoque del CTA; no obstante, muchos de ellos están dirigidos al análisis de quienes juegan un rol profesional en el sistema, específicamente desde la enfermería y la medicina y poco se sabe, desde esta óptica, en relación a otro tipo de tareas y actividades, en particular aquellas que son realizadas por el mismo paciente (Holden et al., 2020). Esto resulta esencial si se considera que el proceso de desarrollo y mejora, desde la E/FH, tiene como punto central el bienestar del paciente, por lo que se hace muy importante indagar sobre cuáles son los recursos cognitivos, el conocimiento y la información que el paciente usa para realizar las actividades que, dentro del proceso de cuidado de su salud, queda en sus manos.

\section{Cambio de tareas (Task shifting)}

Esta tercera herramienta, más que una forma de análisis de las tareas que componen el trabajo, puede definirse como una estrategia que puede contribuir a mejorar los procesos y cumplimiento de los objetivos, a partir de la reasignación de responsabilidades (Aithal \& Aithal, 2017).

El TS (por sus siglas en inglés) consiste en la identificación y posterior transferencia de 
tareas del personal más especializado, al personal menos especializado. Esto es posible a través de un proceso de capacitación y entrenamiento que le otorga al personal menos especializado el conocimiento y habilidades necesarias para encargarse de diferentes tareas, sin que ello represente necesariamente el paso por una educación formal (Aithal \& Aithal, 2017). Dicho proceso tiene como propósito disminuir la carga de trabajo del personal más especializado, y aumentar la eficiencia y eficacia en todos los procesos presentes en estos sistemas, cuando el déficit de personal calificado es muy alto.

La Organización Mundial de la Salud (WHO, 2007), ha establecido cinco categorías y a partir de ellas 22 recomendaciones, como soporte para desarrollar el proceso de implementación del cambio de tareas para que se pueda ajustar a diversas condiciones (tabla 3). Vale señalar que estas recomendaciones tienen como propósito instar a los gobiernos a que asuman estrategias nacionales de mejora. No obstante, muchas de estas especificaciones pueden reconocerse como pasos dentro de las mismas organizaciones, que pueden contribuir a mejorar los sistemas de salud.

Tabla 3. Sugerencias de la Organización Mundial de la Salud (WHO, 2007) para la implementación de un TS.

\begin{tabular}{|c|c|}
\hline Categorías & Recomendaciones específicas \\
\hline $\begin{array}{l}\text { Recomendaciones } \\
\text { para el TS como } \\
\text { iniciativa de salud } \\
\text { pública }\end{array}$ & $\begin{array}{l}\text { 1. Los países, en colaboración con todos los involucrados deberían } \\
\text { considerar la implementación y el mejoramiento del TS para } \\
\text { mejorar las condiciones de la prestación del servicio de salud. } \\
\text { 2. Debe haber un claro esfuerzo por identificar e incluir en el } \\
\text { proceso de adopción del TS, a los involucrados más pertinentes, } \\
\text { incluyendo a los pacientes. } \\
\text { 3. Los países deben esforzarse por implementar esta iniciativa como } \\
\text { una estrategia nacional, bajo una estructura compartida. } \\
\text { 4. Realizar un análisis y actualizar la información sobre los recursos } \\
\text { humanos con los que cuenta el sistema.. }\end{array}$ \\
\hline $\begin{array}{l}\text { Recomendaciones } \\
\text { sobre la creación de } \\
\text { un entorno } \\
\text { normativo adecuado } \\
\text { para la } \\
\text { implementación del } \\
\text { TS }\end{array}$ & $\begin{array}{l}\text { 5. Considerar el uso de enfoques regulatorios que permitan que el } \\
\text { personal pueda ampliar su área de acción y práctica y crear } \\
\text { nuevos grupos de trabajadores. } \\
\text { 6. Considerar una estrategia de revisión rápida para analizar el } \\
\text { sistema regulatorio y hacer los ajustes necesarios para facilitar la } \\
\text { implementación del TS. }\end{array}$ \\
\hline $\begin{array}{l}\text { Recomendaciones } \\
\text { para garantizar la } \\
\text { calidad de la atención }\end{array}$ & $\begin{array}{l}\text { 7. Ajustar los mecanismos de garantía de la calidad de la atención a } \\
\text { los posibles nuevos escenarios que plantea la implementación del } \\
\text { TS. } \\
\text { 8. Establecer roles y competencias estandarizadas para la } \\
\text { ampliación de la acción y práctica de los trabajadores, que sean la } \\
\text { base para su reclutamiento, entrenamiento y evaluación. } \\
\text { 9. Sistematizar y estandarizar los procesos de capacitación y } \\
\text { formación. } \\
\text { 10. Generar programas de entrenamiento y educación continua. } \\
\text { 11. Proporcionar supervisión de apoyo y tutoría constante, } \\
\text { garantizando que quienes proveen dicha supervisión y tutoría, } \\
\text { cuentan con las capacidades profesionales requeridas. } \\
\text { 12. Asegurar que todos los colectivos de trabajadores puedan ser } \\
\text { evaluados a partir de un conjunto de roles, niveles de } \\
\text { competencia y estándares. }\end{array}$ \\
\hline
\end{tabular}




\begin{tabular}{|l|l|}
\hline $\begin{array}{l}\text { Recomendaciones } \\
\text { para garantizar la } \\
\text { sostenibilidad }\end{array}$ & $\begin{array}{l}\text { 13. Considerar medidas de carácter financiero y no financiero, como } \\
\text { incentivo para mantener y mejorar las capacidades de los } \\
\text { trabajadores en sus nuevos roles }\end{array}$ \\
14. Considerar que un buen servicio de salud no puede sostenerse a \\
largo plazo, con personal que trabaja de forma voluntaria.. \\
15. Asegurarse que la implementación de TS tenga un sistema de \\
financiamiento robusto y a largo plazo.
\end{tabular}

\section{Tecnología y herramientas}

La gran mayoría de actividades humanas involucra la mediación de un artefacto o producto que contribuye a obtener los objetivos y mejorar los resultados. En el contexto de la salud, la tecnología ha jugado un papel esencial en los procesos de atención a los pacientes, tanto en actividades completamente médicas como en aspectos del servicio como la comunicación, el seguimiento, o incluso en los procesos de autocuidado. Esto hace necesario el robustecimiento de los recursos tecnológicos, no solo desde la perspectiva técnica, sino, desde la interacción que tienen los involucrados con dichos recursos y la búsqueda de elementos tecnológicos cada vez más fáciles de usar y cada vez más a prueba de errores. A continuación, se presentan tres herramientas de análisis tecnológico, dos de ellas dentro del área de la usabilidad, enfocadas en evaluar la interacción entre seres humanos y tecnología; que pueden contribuir en el mejoramiento de estos elementos y en general en el mejoramiento de los sistemas de salud.

\section{Análisis de enlaces (Link Analysis)}

El LA (por sus siglas en inglés) es un método que permite identificar las relaciones y asociaciones entre los componentes de un sistema, a lo largo de un proceso. En particular, en $\mathrm{E} / \mathrm{FH}$, contribuye a reconocer la interacción entre las personas involucradas en el proceso y todos los recursos y la tecnología a lo largo de una tarea; o entre los involucrados y una 
máquina o artefacto específico (Lin et al., 2008). El análisis de enlaces nos da una perspectiva del movimiento y los puntos de contacto del o los involucrados en la realización de la tarea, lo que permite identificar la frecuencia de la interacción, los elementos centrales o de mayor interacción y las rutas o recorridos en dicha interacción (Lin \& Wu, 2010). Lo anterior es básico para eficientar el proceso, reconociendo un nuevo orden u organización para cumplir los objetivos.

Para llevar a cabo un LA se podrían mencionar cuatro pasos específicos, basados en lo mencionado por Cullinane (1977): a) Elaborar un diagrama representando los elementos del sistema y donde se señalen las conexiones. b) Examinar las conexiones y establecer valores a las conexiones (estos valores pueden estar definidos por la frecuencia o, en aspectos más cualitativos establecidos previamente por el equipo de investigación). c) Desarrollar un diagrama preliminar de conexiones. d) Refinar el diagrama de conexiones y elaborar un diseño final de la interfaz o tarea. Vale señalar que para el proceso de construcción del LA, la forma de recopilación de información será esencial y se sugiere como herramienta básica, la observación y el registro de las tareas.

El LA, tiene la ventaja de poder ser aplicado en diferentes escalas y contextos. Desde el posible análisis de la interacción de un usuario con producto, máquina o artefacto, pasando por el análisis de la interacción de uno o varios involucrados en un espacio particular, hasta el análisis de los enlaces entre personal o involucrados en un entorno o tarea específica. En la figura 2 y tabla 4 se muestran la forma de representación de las rutas y frecuencias respectivamente.

Figura 2. 1. Representación de los puntos de contacto de un usuario en un panel de controles (plano de base tomado de https://ca.dhgate.com/product/arcade-game-joystick-for-pc-for-ps4for-xbox/40671680o.html. 2. Representación de los puntos de contacto de un involucrado con los elementos presentes en un quirófano (plano de base tomado de: https://documentos.arq.com.mx/detalles/201708.html).

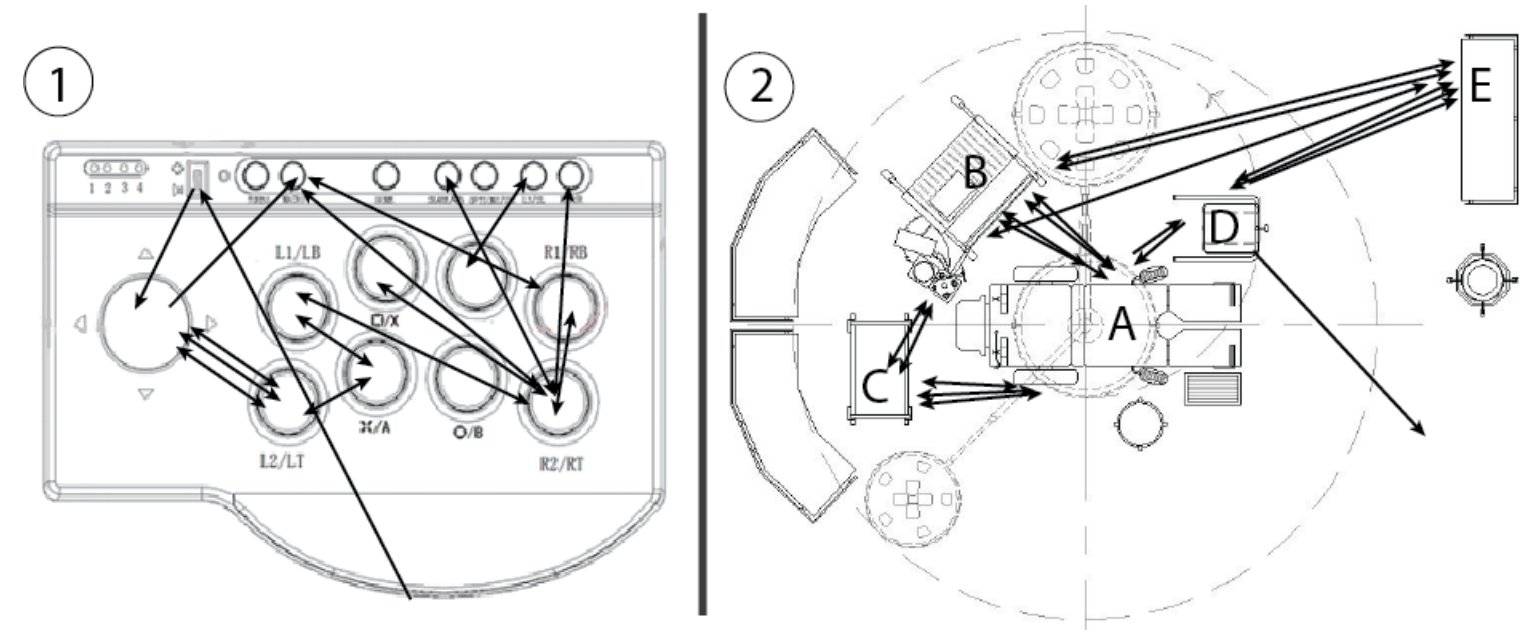


Tabla 4. Ejemplo del reporte de la frecuencia de las conexiones entre equipo médico y ubicación en un compartimiento de ambulancia.

\begin{tabular}{|l|c|c|c|c|c|c|c|c|}
\hline & $\mathbf{1}$ & $\mathbf{2}$ & $\mathbf{3}$ & $\mathbf{4}$ & $\mathbf{5}$ & $\mathbf{6}$ & $\mathbf{7}$ & $\mathbf{8}$ \\
\hline (1)Asiento de atención & & & & & & & & \\
\hline (2)Camilla & 263 & 6 & & & & & & \\
\hline (3)Desfibrilador & 32 & 54 & & & & & & \\
\hline (4)Oxímetro de pulso & 23 & 41 & 7 & & & & & \\
\hline $\begin{array}{l}\text { (5)Superficie de trabajo del banco } \\
\text { delantero }\end{array}$ & 29 & 35 & 2 & 1 & & & & \\
\hline (6)Caja del técnico & 2 & 4 & 1 & & 2 & & & \\
\hline $\begin{array}{l}\text { (7)Caja de medicamentos del } \\
\text { vehículo }\end{array}$ & 15 & 1 & 2 & & & & & \\
\hline (8)Bolsa de resucitación & 1 & 8 & 1 & & & 2 & & 6 \\
\hline
\end{tabular}

Nota. Fragmento tomado de Ferreira y Hignett (2004).

El LA, por estar enfocado en el proceso, pero teniendo como base el análisis de los medios a través de los cuales se realizan las tareas, es una herramienta que media entre el campo de las tareas, descrito desde el modelo SEIPS y el campo de la tecnología. Esto resulta muy importante en caso que se quiera realizar un análisis mixto (Ferreira \& Hignett, 2005) dentro de un contexto de la salud, para tener una mayor claridad de los aspectos que intervienen en los procesos.

\section{Usabilidad... a través de la eficiencia, eficacia y satisfacción}

La usabilidad se define como la propiedad de los sistemas que permiten su fácil uso (Jordan, 1998). Se enfoca en el ajuste de los sistemas a las capacidades y expectativas de los usuarios o involucrados. Existen diversas dimensiones que permiten definir la usabilidad, como la facilidad de aprendizaje, la reducción de los errores, la eficiencia, la facilidad de recordación, la satisfacción, la accesibilidad entre otros (Jacob Nielsen, 1993; Rubin \& Chisnell, 2008). No obstante, los aspectos más básicos de evaluación se podrían concretar en tres: a) Eficiencia, que se define como el uso de la menor cantidad de recursos para conseguir los objetivos de las tareas. b) Eficacia, que se reconoce en relación al grado de consecución de los objetivos y a los errores que se pueden suceder durante la interacción. c) Satisfacción, que se entiende como el nivel de confort que sienten los usuarios en su interacción con el o los sistemas con los que interactúa (Jordan, 1998).

Las herramientas y para realizar un análisis de usabilidad, enfocado en los tres elementos básicos, permiten identificar el tiempo que requieren los involucrados para completar las tareas, el número y naturaleza de los errores en la interacción con los elementos tecnológicos, la tasa de consecución de los objetivos y el confort y la aceptación que tienen del sistema después de haber interactuado con él. En la tabla 5 se presenta una posible secuencia de análisis de usabilidad de un sistema. 
Tabla 5. Secuencia de aplicación de una evaluación de usabilidad.

\begin{tabular}{|c|c|c|}
\hline Paso & Actividad & Herramientas \\
\hline $\begin{array}{l}\text { Identificación y } \\
\text { definición de las tareas a } \\
\text { analizar }\end{array}$ & $\begin{array}{l}\text { Conocer el contenido de la actividad e } \\
\text { identificar los elementos presentes en la } \\
\text { interacción, con el fin de definir los } \\
\text { momentos o tareas a analizar. }\end{array}$ & $\begin{array}{l}\text {-Observación/registro } \\
\text { en video } \\
\text {-Entrevistas semi- } \\
\text { estructuradas }\end{array}$ \\
\hline Descripción de las tareas & $\begin{array}{l}\text { Describir la secuencia de la interacción y } \\
\text { todos aquellos elementos presentes en el } \\
\text { proceso. }\end{array}$ & $\begin{array}{l}\text {-Árbol de tareas } \\
\text {-Diagrama de flujo }\end{array}$ \\
\hline $\begin{array}{l}\text { Definición de los } \\
\text { tiempos de referencia } \\
\text { para la evaluación }\end{array}$ & $\begin{array}{l}\text { Establecer los tiempos de referencia para } \\
\text { evaluar la tarea, a través del análisis de } \\
\text { información preexistente sobre la actividad } \\
\text { y la observación de la realización de la tarea } \\
\text { por un experto. }\end{array}$ & $\begin{array}{l}\text {-Análisis de } \\
\text { documentación } \\
\text {-Observación }\end{array}$ \\
\hline $\begin{array}{l}\text { Definición del tipo de } \\
\text { errores que serán } \\
\text { considerados en el } \\
\text { análisis y posible } \\
\text { número de errores } \\
\text { tolerado en caso que } \\
\text { esto sea factible }\end{array}$ & $\begin{array}{l}\text { Según la naturaleza de la interacción, } \\
\text { definir las acciones del usuario/involucrado } \\
\text { que puedan ser consideradas como un error } \\
\text { en la realización de la tarea. }\end{array}$ & $\begin{array}{l}\text {-Referencias teóricas } \\
\text {-Taxonomías del error }\end{array}$ \\
\hline $\begin{array}{l}\text { Registro de tiempo de } \\
\text { realización por tarea }\end{array}$ & $\begin{array}{l}\text { Medir el tiempo que se toman diferentes } \\
\text { usuarios para completar el propósito de la } \\
\text { tarea. Ideal contar con involucrados } \\
\text { expertos y novatos para identificar } \\
\text { diferencias. Estos tiempos se comparan con } \\
\text { los tiempos de referencia para establecer la } \\
\text { eficiencia. }\end{array}$ & $\begin{array}{l}\text {-Observación/registro } \\
\text { en video } \\
\text {-Formato de registro de } \\
\text { tiempos por usuario y } \\
\text { tarea }\end{array}$ \\
\hline $\begin{array}{l}\text { Registro de tasa de } \\
\text { cumplimiento por tarea }\end{array}$ & $\begin{array}{l}\text { Identificar el número de personas que } \\
\text { completan satisfactoriamente la tarea. Ideal } \\
\text { contar con involucrados expertos y novatos. }\end{array}$ & $\begin{array}{l}\text { - Observación/registro } \\
\text { en video } \\
\text {-Formato de registro de } \\
\text { tasa de cumplimiento } \\
\text { por usuario y tarea }\end{array}$ \\
\hline $\begin{array}{l}\text { Identificación de } \\
\text { número y tipo de errores }\end{array}$ & $\begin{array}{l}\text { Identificar el número de errores y la } \\
\text { naturaleza de los mismos para reconocer } \\
\text { puntos problemáticos. Estos errores se } \\
\text { comparan con lo establecido como } \\
\text { referencia. }\end{array}$ & $\begin{array}{l}\text {-Observación/registro } \\
\text { en video } \\
\text {-Formato de registro de } \\
\text { número y tipo de } \\
\text { errores por usuario y } \\
\text { tarea } \\
\text {-Entrevista }\end{array}$ \\
\hline $\begin{array}{l}\text { Identificar el grado de } \\
\text { satisfacción del } \\
\text { involucrado en la } \\
\text { interacción con el } \\
\text { sistema }\end{array}$ & $\begin{array}{l}\text { Aplicar un cuestionario posterior a cada } \\
\text { tarea para medir el nivel de satisfacción que } \\
\text { pueda tener el involucrado en relación con } \\
\text { el proceso realizado. }\end{array}$ & $\begin{array}{l}\text {-ACQ (After Scenario } \\
\text { Questionnaire) } \\
\text {-PSSUQ (Post-Study } \\
\text { System Usability } \\
\text { Questionnaire) } \\
\end{array}$ \\
\hline $\begin{array}{l}\text { Desarrollo de } \\
\text { recomendaciones de } \\
\text { mejora }\end{array}$ & $\begin{array}{l}\text { Propuesta de mejora de los elementos que } \\
\text { se identificaron como factores negativos en } \\
\text { la usabilidad del sistema. }\end{array}$ & $\begin{array}{l}\text {-Grupos de enfoque } \\
\text { con usuarios y expertos } \\
\text {-Opiniones de expertos } \\
\text {-Teoría }\end{array}$ \\
\hline Pruebas formativas & $\begin{array}{l}\text { Se realizan evaluaciones con pasos similares } \\
\text { a los descritos anteriormente, generalmente } \\
\text { bajo ambientes más controlados para } \\
\text { identificar posibles ajustes antes de su } \\
\text { implementación. }\end{array}$ & $\begin{array}{l}\text {-Protocolos de tareas } \\
\text {-Prototipos }\end{array}$ \\
\hline
\end{tabular}


Si bien, ya hay bastante camino recorrido desde la usabilidad en áreas como el desarrollo de dispositivos médicos (Wiklund, 1995), se considera que el reconocerla como la propiedad de un sistema amplía sus posibles espacios de aplicación y de desarrollo en el terreno de los sistemas de salud.

\section{Usabilidad a través de Heurísticos}

La evaluación Heurística dentro de la usabilidad es un método de análisis a través del cual se busca identificar la facilidad de uso de un sistema, partiendo de un conjunto de conceptos de referencia denominados heurísticos (Nielsen \& Molich, 1990). Es un método dentro de la categoría de los métodos de inspección, que puede ser aplicado por personas con conocimiento básico de usabilidad, con resultados óptimos, pero que funciona de manera muy eficiente con expertos en usabilidad (Nielsen, 1992).

Los heurísticos son especialmente importantes para hacer un acercamiento a los problemas que presentan los elementos tecnológicos en su forma de comunicación e interacción con el usuario o involucrados; con el fin de detectar áreas de oportunidad para corrección y mejora de una manera más simple y sin las implicaciones que tienen las pruebas con usuarios (Chan et al., 2012).

Zhang et al. (2003) propusieron, basados en los heurísticos básicos propuestos por Molich y Nielsen, y por Shneiderman, un conjunto de 14 heurísticos para la evaluación de dispositivos médicos (tabla 6).

Tabla 6. Descripción de los Heurísticos propuestos por Zhang et al. (2003).

\begin{tabular}{|l|l|}
\hline Heurístico & Descripción \\
\hline Consistencia & $\begin{array}{l}\text { El sistema debe evitar usar varios términos para lo mismo o un solo } \\
\text { término para elementos diferentes. También debe tener lenguajes } \\
\text { similares con otros sistemas en caso que estén relacionados. }\end{array}$ \\
\hline Visibilidad & $\begin{array}{l}\text { El estado del sistema y su funcionamiento debe ser visible para el usuario. } \\
\text { modelo mentanto del sistema debe coincidir con las expectativas o el }\end{array}$ \\
\hline Conexión & $\begin{array}{l}\text { Cualquier información extraña o de más genera distracción y retrasos en } \\
\text { las interacción. }\end{array}$ \\
\hline Minimalismo & $\begin{array}{l}\text { El sistema debe minimizar la carga para la memoria del usuario. Evitar } \\
\text { tareas que requieran mantener información de tareas previas. }\end{array}$ \\
\hline Memoria & $\begin{array}{l}\text { El sistema debe proveer de información oportuna y clara según el estado } \\
\text { del mismo. }\end{array}$ \\
\hline Retroalimentación & $\begin{array}{l}\text { Algunas características que resultan importantes para un usuario novato, } \\
\text { como las descripciones prolijas o secuencias de interacción claras, pueden } \\
\text { resultar engorrosas para un usuario con más experiencia, por lo que } \\
\text { proveer de atajos al sistema o sistemas de ajuste al usuario, puede darle al } \\
\text { experto una mejor experiencia de uso. }\end{array}$ \\
\hline Flexibilidad & $\begin{array}{l}\text { Los mensajes de error deben enfocarse en mostrar que el problema está } \\
\text { en el sistema y no en el usuario, debe mostrarle con precisión al usuario } \\
\text { en qué consiste el problema y debe darle información o pistas de cómo } \\
\text { resolverlo. }\end{array}$ \\
\hline Mensaje & $\begin{array}{l}\text { Más que un buen sistema de reporte de error, el sistema debe estar lo } \\
\text { suficientemente bien diseñado para evitar que aparezca el error. }\end{array}$ \\
\hline Error & $\begin{array}{l}\text { Cada tarea debe tener un comienzo y un final y este último debe ser } \\
\text { claramente notificado. }\end{array}$ \\
\hline Cierre & El sistema nunca debe "capturar" a un usuario en una situación en la que \\
\hline Deshacer &
\end{tabular}




\begin{tabular}{|l|l|}
\hline & $\begin{array}{l}\text { no vea una salida clara. Debe permitir que el usuario se equivoque sin que } \\
\text { ello signifique una situación sin retorno. Permitir acciones de regreso o } \\
\text { corrección del error. }\end{array}$ \\
\hline Lenguaje & El lenguaje del sistema debe ser siempre entendible para el usuario. \\
\hline Control & $\begin{array}{l}\text { El sistema debe evitar que el usuario tenga la impresión de ser controlado } \\
\text { por el sistema. }\end{array}$ \\
\hline Ayuda & Siempre proveer ayuda cuando el usuario lo requiera. \\
\hline
\end{tabular}

La evaluación se realiza, dando un nivel de severidad a cada tarea con problemas identificados en relación a cada heurístico (Zhang et al., 2003) (tabla 7). Cabe resaltar que estos niveles de puntuación están enfocados completamente en la usabilidad, por lo que no contemplan un impacto hacia los demás componentes o en relación al cuidado de la salud de los pacientes. Frente a esto, (Cassano-Piché et al., 2015) proponen una puntuación que complementa la escala de severidad de Zhang et al. (2003) con una escala de severidad que integra el concepto de seguridad (tabla 8).

Tabla 7. Niveles de severidad propuestos por Zhang et. al (2003).

\begin{tabular}{|c|l|}
\hline Puntuación & Descripción \\
\hline o & No tiene un problema de usabilidad. \\
\hline 1 & $\begin{array}{l}\text { Es un problema “cosmético". No necesita ser reparado a menos que se cuente } \\
\text { con suficiente tiempo para ello. }\end{array}$ \\
\hline 2 & $\begin{array}{l}\text { Problema menor de usabilidad. El problema tiene una baja prioridad para } \\
\text { ser resuelto. }\end{array}$ \\
\hline 3 & $\begin{array}{l}\text { Problema mayor de usabilidad. El problema tiene una alta prioridad para ser } \\
\text { resuelto. }\end{array}$ \\
\hline 4 & $\begin{array}{l}\text { Catástrofe de usabilidad. Es esencial resolver el problema antes de que llegue } \\
\text { a ser usado en un contexto real. }\end{array}$ \\
\hline
\end{tabular}

Tabla 8. Niveles de severidad relacionados con la usabilidad y la seguridad propuestos por Cassano-piché et al. (2015).

\begin{tabular}{|c|l|}
\hline Puntuación & Descripción \\
\hline 1 & $\begin{array}{l}\text { Severidad baja. El problema puede ser medianamente frustrante para el } \\
\text { usuario. }\end{array}$ \\
\hline 2 & $\begin{array}{l}\text { Severidad media. Un problema serio que puede ser muy frustrante para el } \\
\text { usuario, pero que además dificulta que complete la tarea y que lo haga de } \\
\text { manera eficiente. }\end{array}$ \\
\hline 3 & $\begin{array}{l}\text { Severidad alta. Un problema crítico que disminuye de manera clara la } \\
\text { habilidad del usuario para interactuar con el sistema o que tiene el potencial } \\
\text { de generar un daño en el paciente. }\end{array}$ \\
\hline
\end{tabular}

\section{Discusión}

Entendiendo la condición sistémica de muchas de las problemáticas y puntos de mejora identificados en los escenarios relacionados con la salud, un punto a destacar es la capacidad presente en las distintas herramientas descritas en este documento, para relacionar diversas áreas descritas en el modelo SEIPS 3.0, como es el caso de los HTA o de los análisis de usabilidad. A esto hay que sumar el uso de otras alternativas metodológicas como los mapas de trayecto (journey maps) (Gibbons, 2018) o el service design blueprint (Aceves-González, 2015), que pueden brindar una mirada más holística, a partir de una visualización de los 
elementos presentes en el sistema y su forma de relacionarse. Es importante mencionar que la presencia de la ergonomía en los sistemas de salud no se agota en las herramientas presentes en este texto y que es posible encontrar tanto para la evaluación de las tareas, como para la evaluación de los recursos tecnológicos, otras opciones metodológicas relevantes para ser consideradas (Holden \& Carayon, 2021).

\section{Conclusiones}

El bienestar del paciente debe ser el punto de anclaje de cualquier iniciativa de mejora de los sistemas de salud. Para ello se hace indispensable tener alternativas de análisis e intervención que contribuyan a mejorar las interacciones presentes en estos escenarios de gran complejidad. Bajo esta idea, es importante seguir en el proceso de acercamiento y adaptación de herramientas de la ergonomía dentro de los sistemas de salud, para robustecer sus procesos, su presencia y su contribución en la búsqueda de sistemas de mejor calidad. 


\section{Referencias}

Aceves-González, C. (2015). The application and development of inclusive service design in the context of a bus service (Issue May). Loughborough University.

Aithal, A., \& Aithal, P. S. (2017). ABCD An nalysis of Task Shifting - An optimum Alternative Sollution to Professioonal Healthcare Personnel Shortage. International Journal of Health Sciences and Pharmacy, 1(2), 36-51.

Al-Assaf, A. F., Bumpus, L. J., Carter, D., \& Dixon, S. B. (2003). Preventing errors in healthcare: a call for action. Hospital Topics, 81(3), 5-13. https://doi.org/10.1080/00185860309598022

Carayon, P., Schoofs Hundt, A., Karsh, B.-T., Gurses, A. ., Alvarado, C. ., Smith, M., \& Flatley Brennan, P. (2006). Work system design for patient safety : the SEIPS model. BMJ Quality \& Safety, 15, 50-59. https://doi.org/10.1136/qshc.2005.015842

Carayon, Pascale, \& Wood, K. E. (2010). Patient Safety: The Role of Human Factors and Systems Engineering. Stud Health Technol Inform, 153, 23-46.

Carayon, Pascale, Wooldridge, A., Hoonakker, P., Hundt, A. S., \& Kelly, M. M. (2020). SEIPS 3.0: Human-centered design of the patient journey for patient safety. Applied Ergonomics, 84(December 2018), 103033. https://doi.org/10.1016/j.apergo.2019.103033

Carayon, Pascale, Xie, A., \& Kianfar, S. (2014). Human factors and ergonomics as a patient safety practice. BMJ Quality \& Safety, 23, 196-205. https://doi.org/10.1136/bmjqs-2013-001812

Cassano-Piché, A., Trbovich, P., Griffin, M., Ling Lin, Y., \& Easty, T. (2015). Human Factors for Health Technology Safety: Evaluating \& Improving the use of Health Technology in the Real World. http://cedglobal.org/wp-content/uploads/2016/11/CED-HF-HealthTechnology-Safety.pdf

Chan, A. J., Islam, M. K., Rosewall, T., Jaffray, D. A., Easty, A. C., \& Cafazzo, J. A. (2012). Applying usability heuristics to radiotherapy systems. Radiotherapy and Oncology, 102(1), 142-147. https://doi.org/10.1016/j.radonc.2011.05.077

Clark, R., Feldon, D., Van Merrienboer, J. J. G., Yates, K., \& Early, S. (2016). Chapter 43: Cognitive Task Analysis. In Handbook of research on educational communications and technology (pp. 577-593). https://www.researchgate.net/publication/294699964 Cognitive task analysis

Cullinane, T. P. (1977). Minimizing Cost and Effort in Performing a Link Analysis. Human Factors: The Journal of Human Factors and Ergonomics Society, 19(2), 151-156. https://doi.org/10.1177/001872087701900204

Ferreira, J., \& Hignett, S. (2005). Reviewing ambulance design for clinical efficiency and paramedic safety. Applied Ergonomics, 36, 97-105. https://doi.org/10.1016/j.apergo.2004.07.003

Gibbons, S. (2018). Journey Mapping 101. Nielsen Norman Group. https://www.nngroup.com/articles/journey-mapping-101/

Hajdukiewicz, J. R., \& Vicente, K. J. (2004). A theoretical note on the relationship between work domain analysis and task analysis. Theoretical Issues in Ergonomics Science, 5(6), 527-538. https://doi.org/10.1080/146392204123313003427

Holden, R., Carayon, P., Gurses, A., Hoonakker, P., Schoofs Hundt, A., Ozok, A., \& RiveraRodriguez, J. (2013). SEIPS 2.0: A human factors framework for studying and improving the work of healthcare professionals and patients Richard. Ergonomics, 56(11), 1-30. https://doi.org/10.1080/00140139.2013.838643.SEIPS

Holden, R. J., \& Carayon, P. (2021). SEIPS 101 and seven simple SEIPS tools. BMJ Quality \& Safety, $O$, 1-10. https://doi.org/10.1136/bmjqs-2020-012538 
Holden, R. J., Daley, C. N., Mickelson, R. S., Bolchini, D., Toscos, T., Cornet, V. P., Miller, A., \& Mirro, M. J. (2020). Patient decision-making personas : An application of a patient-centered cognitive task analysis ( P-CTA ). Applied Ergonomics, 87(March), 103107. https://doi.org/10.1016/j.apergo.2020.103107

Jordan, P. (1998). An Introduction to Usability. Taylor \& Francis Ltd.

Jun, G. T., Ward, J., Morris, Z. O. E., \& Clarkson, J. (2009). Health care process modelling : which method when? International Journal for Quality in Health Care, 21(3), 214-224.

Lane, R., Stanton, N. A., \& Harrison, D. (2006). Applying hierarchical task analysis to medication administration errors. Applied Ergonomics, 37(5), 669-679. https://doi.org/10.1016/j.apergo.2005.08.001

Lavanderos, S., Pedraza, J., Russo N., M., \& Salas, S. (2016). Dilemas éticos acerca de la revelación de errores médicos a los pacientes. Revista Médica de Chile, 144(9), 1191-1198.

Lin, C. J., Yu, W., \& Wu, C. (2008). Improving link analysis method in user interface design using a new computational optimization algorithm. Proceedings of the Human Factors and Ergonomics Society, 2, 1112-1116. https://doi.org/10.1177/154193120805201603

Lin, C., \& Wu, C. (2010). Improved link analysis method for user interface design - modified link table and optimisation- based algorithm. Behaviour \& Information Technology, 29(2), 199216. https://doi.org/10.1080/01449290903233892

Nielsen, Jacob. (1993). Usability Engineering. Academic Press.

Nielsen, Jakob. (1992). Finding usability problems through heuristic evaluation. Conference on Human Factors in Computing Systems - Proceedings, 373-380. https://doi.org/10.1145/142750.142834

Nielsen, Jakob, \& Molich, R. (1990). Heuristic evaluation of user interfaces. Conference on Human Factors in Computing Systems - Proceedings, 249-256. https://doi.org/10.1145/97243.97281

Potworowski, G., \& Green, L. A. (2013). Cognitive Task Analysis: Methods to Improve PatientCentered Medical Home Models by Understanding and Leveraging its Knowledge Work. In Agency for Healthcare Research and Quality (AHRQ). http://pcmh.ahrq.gov/sites/default/files/attachments/CognitiveTask 032513comp.pdf

Rasmussen, J. (1986). Information processing and human-machine interaction: An approach to cognitive engineering. In Information Processing \& Management. Elsevier SciencePublishers Co. Inc. https://doi.org/10.1016/0306-4573(88)90080-5

Rubin, J., \& Chisnell, D. (2008). Handbook of Usability Testing How to Plan, Design, and Conduct Effective Tests.

Ruck, H. (1980). Task Analysis Handbook (Issue July).

Salmon, P., Jenkins, D., Stanton, N., \& Walker, G. (2010). Hierarchical task analysis vs. cognitive work analysis: Comparison of theory, methodology and contribution to system design. Theoretical Issues in Ergonomics Science, 11(6), 504-531. https://doi.org/10.1080/14639220903165169

Sarker, S. K., Chang, A., Albrani, T., \& Vincent, C. (2008). Constructing hierarchical task analysis in surgery. Surgical Endoscopy and Other Interventional Techniques, 22(1), 107-111. https://doi.org/10.1007/s00464-007-9380-Z

Singleton, W. T. (1972). Introduction to Ergonomics. World Health Organization.

Stanton, N. A. (2006). Hierarchical task analysis: Developments, applications, and extensions. Applied Ergonomics, 37(1 SPEC. ISS.), 55-79. https://doi.org/10.1016/j.aperg0.2005.06.003

World Health Organization-PatientSafety. (2009). Human Factors in Patient Safety Review of Topics and Tools. 
https://www.who.int/patientsafety/research/methods measures/human factors/human f actors review.pdf

World Health Organization. (2003). The World Health Report 2003. Shaping the future. https://www.who.int/whr/2003/en/whro3 en.pdf

World Health Organization. (2019). 10 datos sobre la seguridad del paciente. Cifras y datos. https://www.who.int/features/factfiles/patient safety/es/

World Health Organization. (2021). Global Patient Safety Action Plan 2021-2030. Towards Eliminating Avoidable Harm in Health Care. https://www.who.int/teams/integratedhealth-services/patient-safety/policy/global-patient-safety-action-plan

Wiklund, M. E. (1995). Medical Device and Equipment Design. Usability Engineering and Ergonomics. CRC Press.

World Health Organization. (2007). Task shifting: rational redistribution of tasks among health workforce teams. https://doi.org/10.1016/s0968-8080(09)33452-7

Zhang, J., Johnson, T. R., Patel, V. L., Paige, D. L., \& Kubose, T. (2003). Using usability heuristics to evaluate patient safety of medical devices. Journal of Biomedical Informatics, 36(1-2), 23-30. https://doi.org/10.1016/S1532-0464(03)00060-1 